\title{
Caring for the caregivers: perception of pharmacists as care providers
}

\author{
Florence Osisanya ${ }^{1 *}$ and Angela C Riley ${ }^{2}$ \\ ${ }^{1}$ College of Pharmacy, Chicago State University, Chicago, Illinois, USA \\ ${ }^{2}$ School of Pharmacy and Pharmaceutical Sciences, Binghamton University, USA
}

\begin{abstract}
Objective: To assess caregivers' perception of pharmacists as care providers and increase awareness of available pharmacy services.

Background: Caregiver roles cover a broad range of expectations/levels of care in the life of the care recipient. The role of the caregiver is constantly evolving, from assistance with basic activities to medical tasks, such as medication management, administration of injections, and wound care. As the level of acuity of care recipients' increase, the responsibilities of the caregiver and number of medications needed increase as well. Caregiver burden is often an overlooked stress which can lead to health challenges for the caregiver and the care recipient. Pharmacists must develop relationships with caregivers to educate and improve their willingness to utilize pharmacy services. The objective of this study was to ascertain caregivers' perception of pharmacists as healthcare providers and educate them on available pharmacy services.
\end{abstract}

Design: A pre-post survey study.

Setting: One church and two senior centers in the Chicagoland area.

Participants: Community dwelling older adults.

Intervention: Upon completion of the pre-survey questions, each participant listened to a 20 min presentation on the role of a caregiver and available pharmacy services. The presentation was interactive and educational. It started off with defining what a caregiver was and ended with educating caregivers about pharmacy services. After the presentation each participant was asked to complete a post-survey.

Results: Forty three completed surveys were returned from participants. Out of these surveys, twenty three respondents were classified as active caregivers. The majority of respondents in the study were female (78.3\%) and Caucasian (56.5\%). An equal group of respondents completed one to three years of college or more than 4 years (39.1\%). The majority of respondents were 55 years or more (89.4\%). Furthermore findings that 1 to 10 hours was the largest group ( 9 respondents). A 5 -point increase was shown in the strongly agree section from pre to post survey (5-10) which was associated with a positive outcome about the perception of pharmacists in the caregiver community.

Conclusion: It was determined that caregivers and care recipients were unaware of the role of the pharmacist, particularly as care providers. The survey also demonstrated positive perception of pharmacists as care providers from caregivers overall. This study demonstrates as caregivers become more aware of available pharmacy services and perceive pharmacists as care providers, they will be a stronger support to the care recipient.

\section{Introduction}

As the generation of baby boomers become elderly, the need for quality care providers to assist in completion of daily needs also increase $[1,2]$. Older adults have become the fastest growing segment of the population and are expected to reach 70 million by year 2030 [3]. Along with this population size, the number adults who will become primary caregivers will increase [3]. An informal caregiver is defined as an unpaid individual involved in assisting others with activities of daily living (ADLs) and or medical tasks. A formal caregiver is a paid care provider providing care in one's home or in a care setting [4]. From the family caregiver, all the way to a formal caregiver, they have been deemed as giving care in one's home or in a care setting. An estimated 42 million Americans serve as caregivers to adult patients and spend 20 hours per week [5]. The role of the caregiver is constantly evolving and becoming increasingly demanding as the level of acuity of those cared for also increases. The primary responsibilities of family caregivers often include assisting their loved ones with ADLs such as bathing, dressing and eating [6]. No matter what the circumstances, the burden of being a caregiver can be overwhelming [3].

Of primary importance to the caregiver is the patient's declining health over time. Gillick's report states that substantial disability characterizes the final year of life in the majority of the elderly population [5]. Falls is an injury that can aid in this disability. A study mentions among people aged 64 and older, 33\% living in community and $50 \%$ living in an institution fall every year [3]. Some of these individuals may experience exacerbations of illness with a need for personal assistance. In addition to providing assistance to prevent falls

*Correspondence to: Florence Osisanya, College of Pharmacy, Chicago State University, Chicago, Illinois, USA, E-mail: florence.osisanya@gmail.com

Key words: pharmacists, transitional care, care providers

Received: January 04, 2017; Accepted: February 02, 2017; Published: February 06, 2017 
and other complications, caregivers focus on psychosocial support for their recipients as well [5]. Individuals who continue to interact with others tend to be healthier, both physically and mentally, than those who are socially isolated [3].

Currently, there is an emphasis on the prevention of chronic diseases in older adults. Responsible for the daily activities of the elderly, caregivers support older adults with chronic or disabling illnesses, but are not included in the processes to prevent disease progression [3]. In order to ensure all older adults are adequately supported with quality providers that are skilled to assist in all areas of need, caregivers must be properly trained and compensated to provide adequate levels of care. Findings revealed in 2010 the caregiver support ratio was more than 7 caregivers for every person in high-risk years of 80 and older. This ratio is projected to decline sharply to 4 caregivers by the year 2030 [7].

The role of family caregivers has dramatically expanded to include performing medical tasks. A national survey reported $46 \%$ of caregivers reported performing medical/nursing tasks such as administering injections and giving 5 to 9 prescription medications daily [6]. Caregivers mentioned these tasks were time consuming, increased their level of anxiety, and caused the number of medication errors to increase for the care recipients. The report further mentioned most care recipients (69\%) did not have home visits by a healthcare professional. This placed stress and worry upon caregivers because they were serving as primary care coordinators without medical expertise [6]. An article stated that nearly half of all caregivers report they are responsible for complex medical tasks that require a professional nurse or trained technician. These tasks included wound care, administering parenteral medications, and operating heavy medical equipment such as mechanical ventilators and dialysis machines [5].

With the older adult population growing, the demand for pharmacists to manage drug therapy will increase [3]. A pharmacist is defined by the American College of Clinical Pharmacy as an individual that provides patient care that optimizes medication therapy and promotes health [8]. Pharmacy services are frequently rendered as part of an interdisciplinary team. Pharmacists play an integral role in coordinating drug management [3]. Problems will be reduced if the pharmacist serves as the medication specialist for the care recipient and caregiver, as demonstrated in a recent study that found 1.5 million adverse effects and medication related problems were prevented through pharmacists driven medication therapy management [9]. Medication management is a specialty that pharmacists are experts at when it comes to the elderly. A case review by Steinman and Hanlon discussed nearly $20 \%$ of community dwelling adults age 65 and older take up to 10 or more medications on a daily basis [10]. Multiple medication use can result in many negative outcomes mainly being adverse drug events. These events are reported as weight loss, falls and decline in functional and cognitive status. The review also mentions 5-35\% of older persons living in a community setting are affected by these events; in which $10 \%$ of these have led to hospital admissions [10].

Pharmacists must develop relationships with caregivers to educate and improve their willingness to utilize pharmacy services. The most common setting for establishing relationships with caregivers, for pharmacists, is in community. Literature reveals many community pharmacies offer chronic disease management services such as diabetes and hypertension, which aid in development of a strong pharmacistpatient (caregiver) relationship [3]. In a study that analyzed the perception of pharmacists in the community setting [11], it was demonstrated that $53 \%$ of respondents have a strong relationship with their pharmacist and $83 \%$ of respondents report feeling respected by their pharmacist; which both reflect positive patient outcomes. A total of 1,031 participants responded to the survey about their overall experience with their community pharmacists and 361 respondents were caregivers.

To further the validity of this literature, a current study was developed amongst the caregiver population. The objective of this study was to ascertain caregivers' perception of pharmacists as care providers and educate them about available pharmacy services to help them effectively care for their loved ones.

\section{Methods}

\section{Study design}

This study design utilized both a pre- post survey combined with an intervention to define and describe the role of the pharmacist. Selection of the study sites was an ongoing process due to the need of the researcher to educate and assess the perception of caregivers. After obtaining approval by the Chicago State University Institutional Review Board, we contacted Senior Centers and Churches within the Chicagoland area. Approval was obtained from one church and two centers resulting in a total of 43 participants.

\section{Participants}

In order to participate each candidate must have been: 18 years or older, actively a caregiver and participated presentation/survey completion. The exclusion criteria was as follows: individuals under the age of 18 years old, physical limitations that may prevent presentation/ survey completion (e.g. visual/hearing/cognitive impairments) and individuals who were not actively providing care to their loved one.

\section{Application of the surveys}

Surveys were coded and stapled together; answers provided by the participants correlated to the code numbers on the surveys. Participants were asked to provide personal demographic data such as gender, race, age, religious background, highest level of education, if they were an active caregiver, and hours spent providing care for their loved one. Using a 5-point Likert scale ranging from "Strongly disagree" to "Does not apply", participants were asked to classify level of quality and education they have received from going to their local pharmacy. Likewise, using a different 5-point Likert scale ranging from "Very likely" to "I don't know", participants were asked to classify which of the following services: Vaccinations, Blood Pressure Monitoring, Blood Glucose Monitoring, Blood Cholesterol Monitoring and Medication Therapy Management would they ask a pharmacist to provide.

Furthermore, participants were asked to classify: where they currently obtain their medications, their perception of the role of a pharmacist, which is to provide patient care that optimizes

medication therapy and promotes health, and how often they speak with a pharmacist.

\section{Intervention}

Upon completion of the pre-survey questions, participants listened to a 20 minute presentation on the role of a caregiver, pharmacist as care providers and pharmacy services available. The presentation was interactive and educational. After the presentation each participant was asked to complete a post-survey. Participation in these surveys was voluntary and no remuneration was provided. Prior to distribution, 
the surveys were reviewed and approved by the Institutional Review Board at Chicago State University.

\section{Study analysis and data collection}

Standardized data was collected and entered into a Microsoft Excel spreadsheet. To analyze variability in responses from pre to post, averages from both surveys were compared. Data was kept and stored away.

\section{Results}

Forty three completed surveys were returned from participants. Of these surveys, twenty three were entirely complete. The majority of respondents in the study were female (78.3\%) and Caucasian (56.5\%). An equal group of respondents completed one to three years of college or more than 4 years $(39.1 \%)$. The majority of respondents were 55 years and older (89.4\%) (Table 1).

The largest number of hours spent providing care for loved ones was 1 to 10 (9 respondents). The lowest group was 21 to 30 hours (2 respondents). The category of 1 to 10 hours was associated with the majority of respondents (Figure 1).

The responses in the strongly agree category improved in from pre

Table 1. Demographic Characteristics of Respondents.

\begin{tabular}{|c|c|}
\hline & No. $(\%)$ \\
\hline \multicolumn{2}{|l|}{ Gender } \\
\hline Males & $5(21.7)$ \\
\hline Females & $18(78.3)$ \\
\hline \multicolumn{2}{|l|}{ Race } \\
\hline African American & $7(30.4)$ \\
\hline Caucasian & $13(56.5)$ \\
\hline Hispanic & $1(4.3)$ \\
\hline Asian & $1(4.3)$ \\
\hline Other & $1(4.3)$ \\
\hline \multicolumn{2}{|l|}{ Education } \\
\hline Grade 12 or GED & $4(17.4)$ \\
\hline College 1 to 3 years & $9(39.1)$ \\
\hline College 4 years or more & $9(39.1)$ \\
\hline \multicolumn{2}{|l|}{ Age (in years) } \\
\hline $45-54$ & $2(10.5)$ \\
\hline 55 or more & $17(89.4)$ \\
\hline
\end{tabular}

to post survey ( 2 points). More than half of the respondents $(65.2 \%)$ strongly agreed that they would feel comfortable going to the pharmacy to fill prescriptions (Figure 2).

The number of respondents in the strongly agree category increased from pre to post survey (4 points). More than half of the respondents $(69.5 \%)$ strongly agreed that they view pharmacists as someone who can offer education about medications (Figure3).

The number of respondents in the strongly agree category increased from pre to post survey ( 1 point increase). More than half of the respondents (56.5\%) would strongly agree they view pharmacists as a valued member of the healthcare team (Figure 4).

The number of responses in the strongly agree category increased from pre to post survey (4-points). More than half of the respondents (56.5\%) would strongly agree they would be open to having the pharmacist embrace more of role with medication management (Figure 5).

The strongly agree category improved from pre to post survey (3 points). More than half of the respondents (65.2\%) would strongly agree they would be open to having the pharmacist monitor conditions and help identify any unmet goals of medication use (Figure 6).

The largest improvement from pre to post survey was the once every 3 months category (5 points). A majority of the respondents (43.5\%) would be willing to speak to a pharmacist once every 3 months (Figure 7).

\section{Discussion}

The main endpoints of the study were to increase awareness/ understanding of pharmacy services amongst caregivers and to assess improvement in the perception of pharmacists as care providers. Demographic characteristics such as level of education (39.1\% finished four or more years of college) proved that respondents were capable of understanding these endpoints. Although the majority of respondents were female (78.3\%) and Caucasian (56.5\%), this did not accurately reflect the community as a whole. Likewise, a smaller group of males (21.7\%), African Americans (30.4\%), Hispanics (4.3\%) and Asians (4.3\%) were not accurately represented. Overall, within the respondent population there was change depicted from pre to post survey.

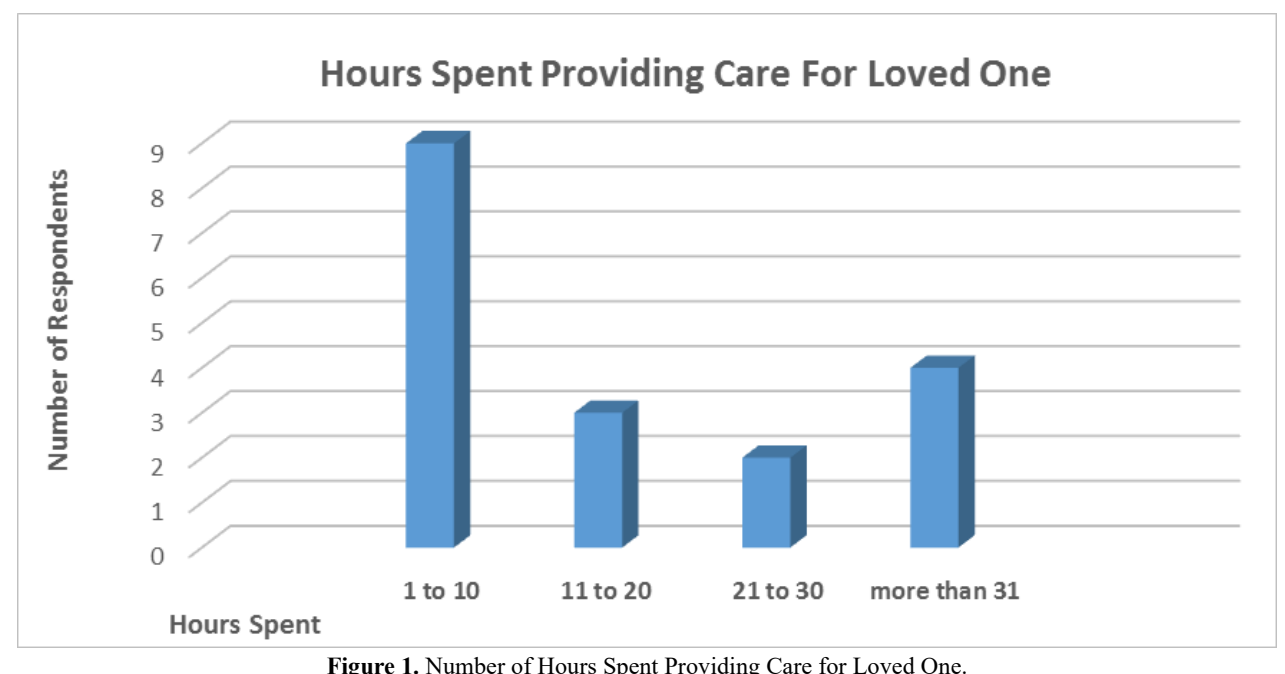

Figure 1. Number of Hours Spent Providing Care for Loved One. 


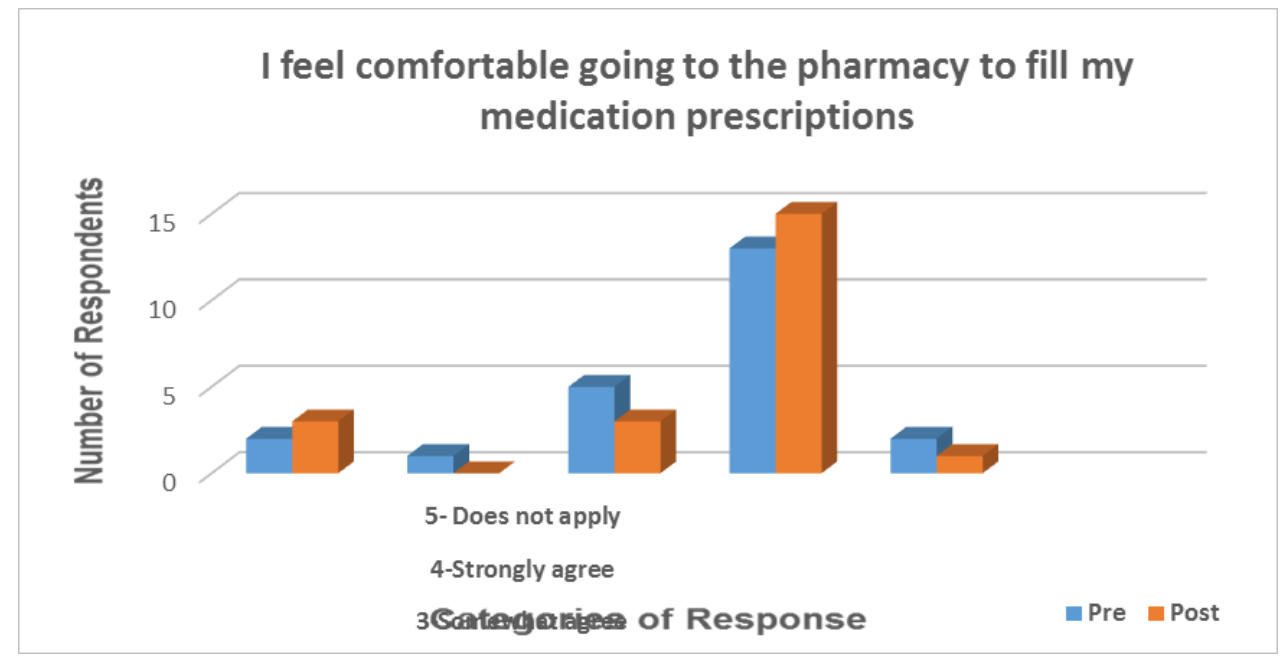

*Based on a 5 -point Likert scale (5=does not apply, $1=$ strongly disagree)

Figure 2. Respondents' Level of Comfortability of Going to the Pharmacy.

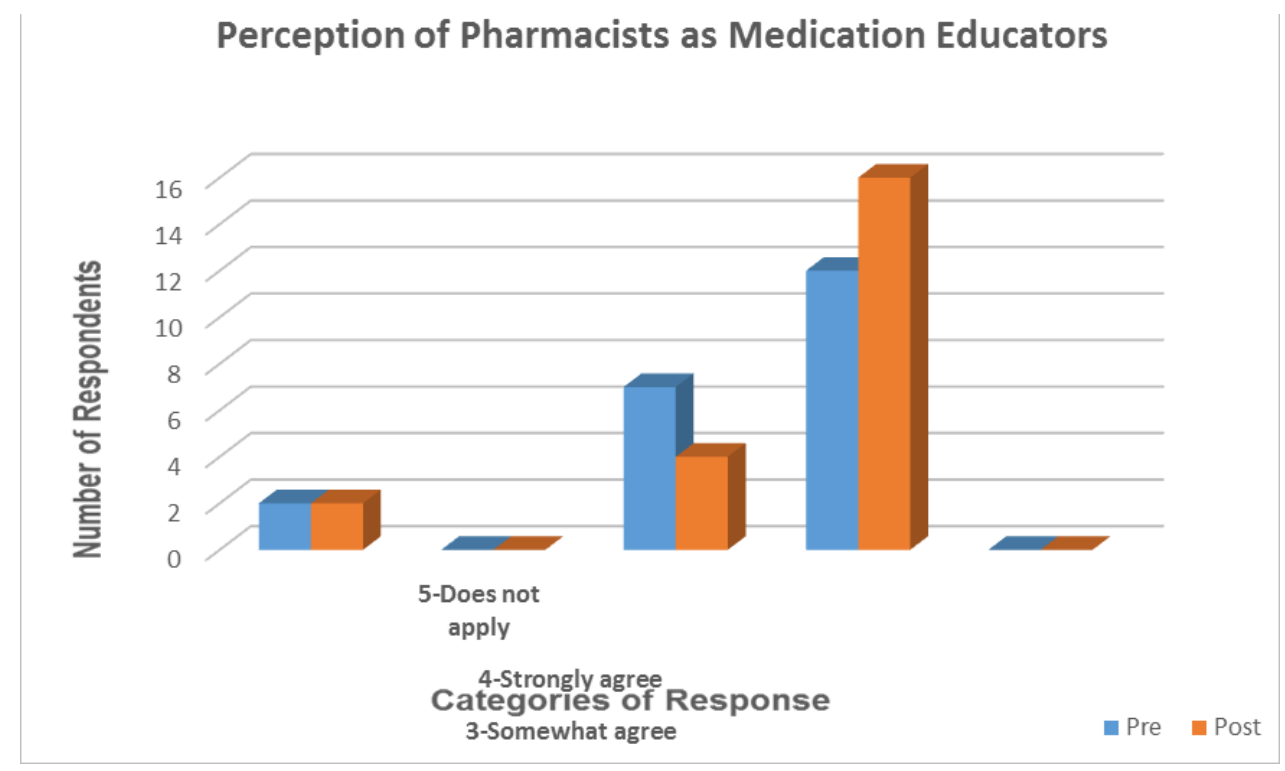

*Based on a 5-point Likert scale ( $5=$ does not apply, $1=$ strongly disagree)

Figure 3. Respondents' Viewing the Pharmacist as Someone Who can Offer Education about Medication I view pharmacists as a value member of the health care team

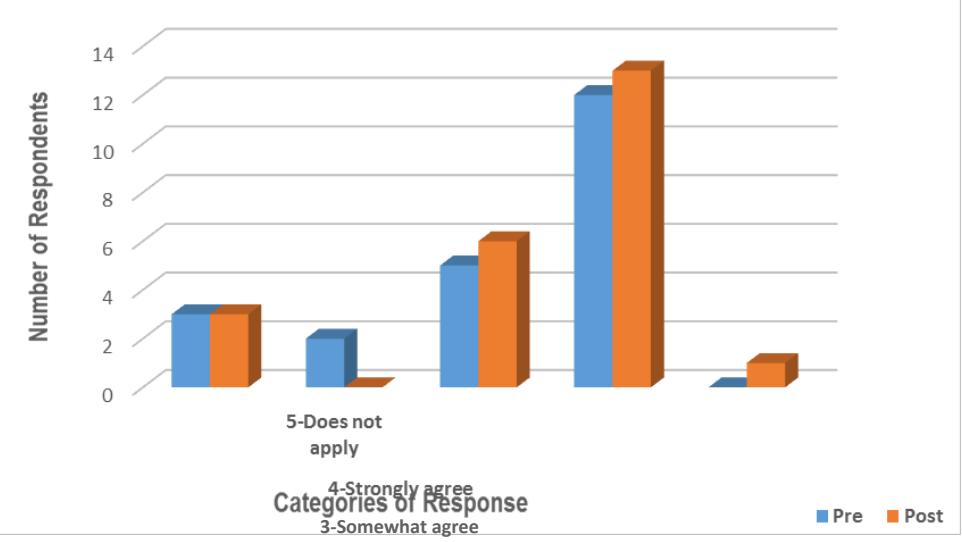

*Based on a 5 -point Likert scale (5=does not apply, $1=$ strongly disagree)

Figure 4. Respondents' Viewing Pharmacists as a Valued Member of the Healthcare Team 


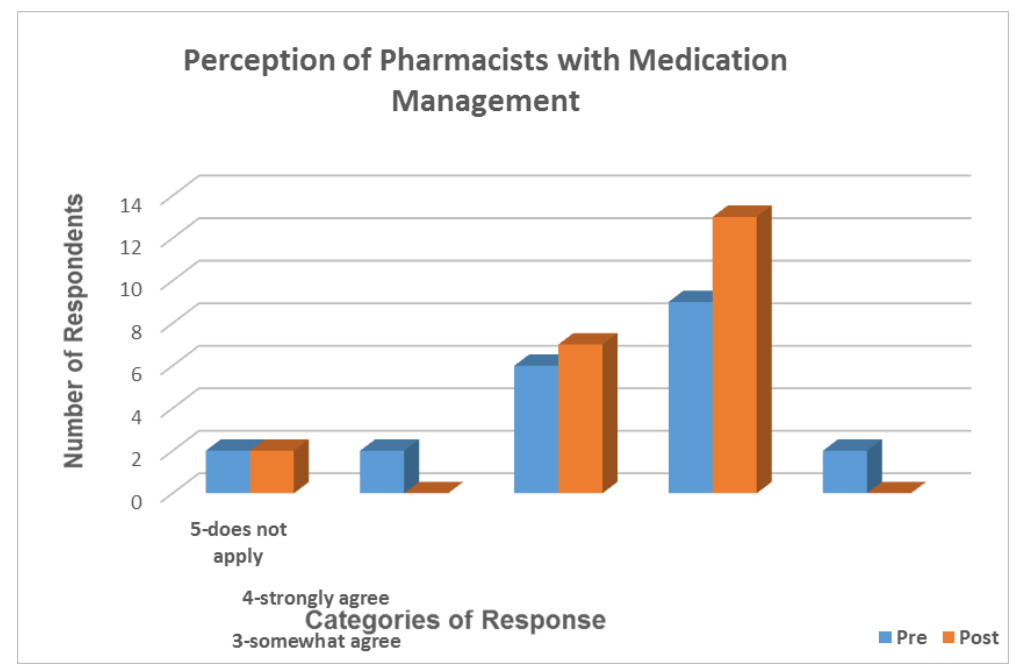

*Based on a 5-point Likert scale ( $5=$ does not apply, $1=$ strongly disagree)

Figure 5. Respondents' Being Open to Having Pharmacists Embrace More of a Role with Medication Management

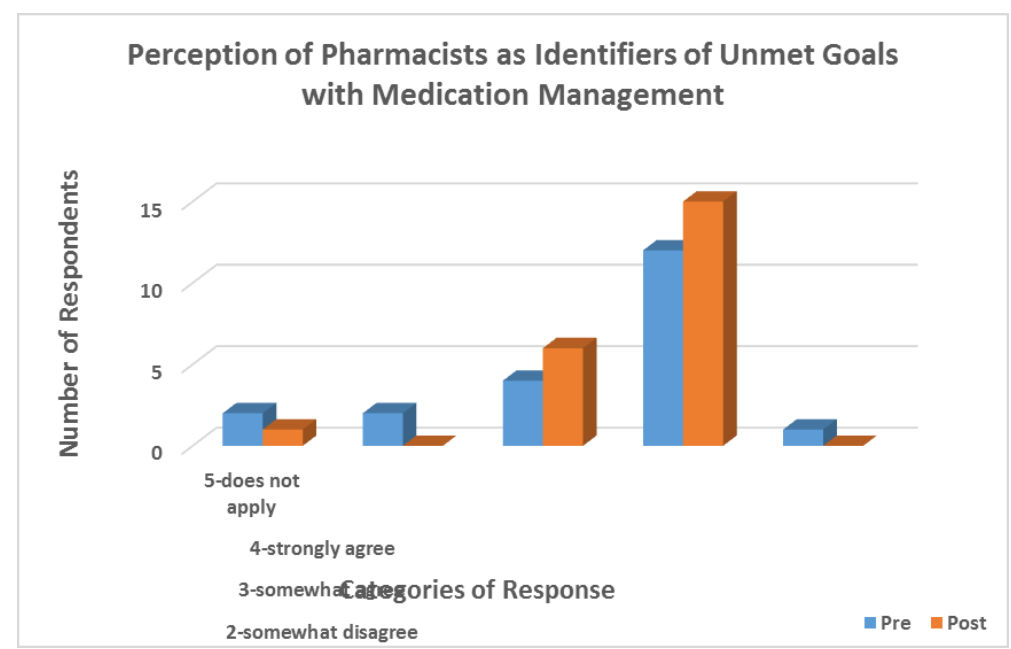

*Based on a 5-point Likert scale (5=does not apply, 1=strongly disagree)

Figure 6. Respondents' Being Open to Having Pharmacist Help Monitor Conditions and Identify any Unmet Goals while Using Medication

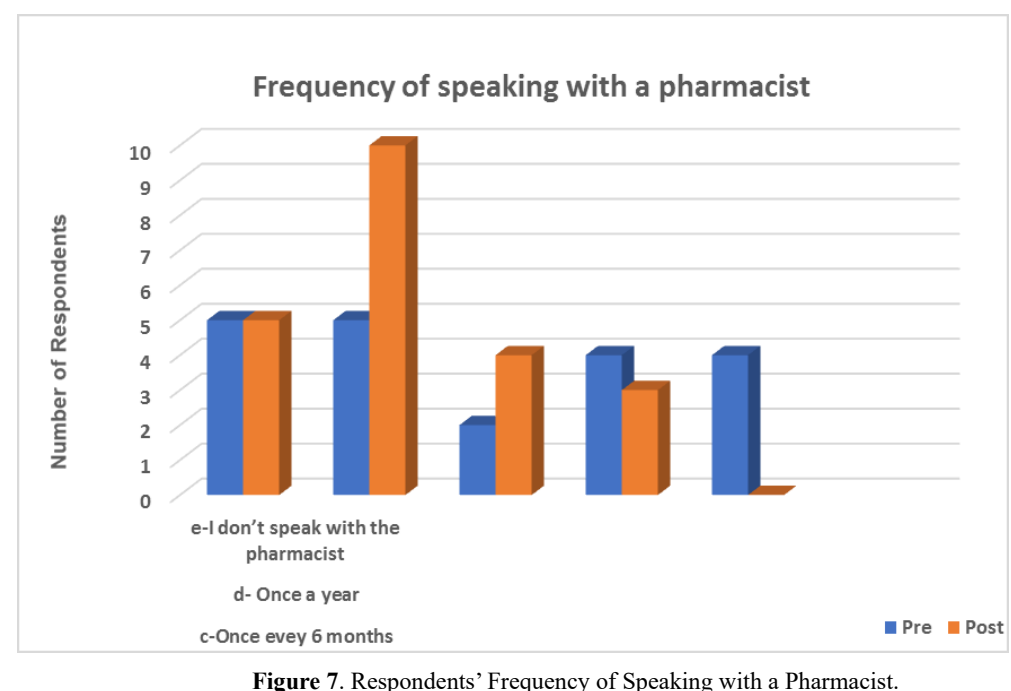

Figure 7. Respondents' Frequency of Speaking with a Pharmacist. 
The first set of questions in the quality section of the survey assessed the level of comfortability of respondents going to the pharmacy and picking up medications. On the pre-survey, 13 of the respondents strongly agreed while the rest selected other options. One comment from a respondent was that every time they go to the pharmacy, they feel the pharmacist is too busy and there is not enough time spent for each person. Other respondents commented in a positive manner. The post-survey showed an increase in the number of responses in the strongly agree category which was and improvement in their perception. Likewise the majority of respondents view pharmacists as medications educators. Initially, the majority of respondents believed education should always come from their doctor. After the presentation, individuals became more aware that pharmacists specialize in medication education and management. One respondent commented on how she relies on her pharmacy first and foremost when it comes to education of her medication. The overall perception of pharmacists from respondents was positive based on responses in this section.

The last question on both pre and post surveys assessed the frequency of speaking to a pharmacist. Improvement was shown in the once every 3 months category from pre to post survey ( 5 points). This was a positive outcome because caregivers are willing to speak more often to a pharmacist than before.

This study had several limitations. First, individuals who were former caregivers were not included in the study. This population could have positively affected the perception of pharmacists based upon their experiences. For example they may have learned the value of the pharmacist because of medication needs, and or concerns formed during the care of their loved one. Secondly, the caregiver's employment status and hours worked were not included in the demographic characteristics. Thirdly, individuals under the age of 18 could not participate in the study. One last limitation included respondents' inability to complete pre/ post surveys, and their personal feelings about participating in research that prevented participation.

\section{Conclusion}

In conclusion, most caregivers are ill prepared for their role. Burdens and health risks will arise from this and will hinder the caregivers from providing effective care [4]. Pharmacy education related to caregivers has improved slightly in the past several years but will need to increase more [3]. The survey that analyzed the perception of pharmacists in the community setting resulted in positive outcomes with mental health patients [11]. To assess this perception amongst the caregiver population, a study was developed. The main purposes of this study was to assess perception of pharmacists as care providers to caregivers; and educate them on available pharmacy services to help them effectively provide care. Analysis of the data showed there was an increase in awareness and education from caregivers about pharmacy services. Likewise, it was found that caregivers have a positive perception of pharmacists based on their answers to survey questions. Although there were many limitations in this study, the main objective was achieved. Due to only twenty-three respondents being classified as caregivers, overall responses in the strongly agree section were minimal. Further research is needed in more caregiver populations to promote awareness through education about pharmacy services.

\section{References}

1. Mehta, Bhavna, and Sweta Parekh. "Caring of Old: Carer's Perspective." International Journal of Social Work and Human Services Practice 4: 104-110.
2. Alonso F, Baró X, Escalera S, Gonzàlez J, MacKay M, Serrahima A (2016) Care respite: taking care of the caregivers. Int J Integrated Care 16: A132.

3. Linnebur SA, O'Connell MB, Wessell AM, McCord AD, Kennedy DH, et al. (2005) Pharmacy Practice, Research, Education and Advocacy for Older Adults. ACCP Pharmacy and the Elderly 25: 1396-1430.[Crossref]

4. Family Caregiver Alliance. Caregiver Statistics: Demographics. National Center on Caregiving 2016. www.caregiver.org/caregiver-statistics-demographics. Accessed January 30th, 2017.

5. Gillick M (2013) The Critical Role of Caregivers in Achieving Patient-Centered Care. JAMA 310: 6. [Crossref]

6. Reinhard S, Levine C, Samis S (2012) Home Alone: Family Caregivers Providing Complex Chronic Care. AARP Public Policy Institute.

7. Redfoot D, Feinberg L, Houser A (2013) The Aging of the Baby Boom and the Growing Care Gap: A Look at Future Declines in the Availability of Family Caregivers. AARP Public Policy Institute.

8. Burke A, Coleman J, Covinsky J et al. American College of Clinical Pharmacy 2014 www.accp.comAccessed on April 13 ${ }^{\text {th }}, 2014$

9. American Pharmacists Association and the National Association of Chain Drug Stores Foundation. Medication Therapy Management in Pharmacy Practice: Core Elements of an MTM Service Model 2008. [

10. Steinman M, Hanlon J (2010) Managing Medications in Clinically Complex Elders "There's Got to Be a Happy Medium". JAMA304: 1592-1601. [Crossref]

11. Caley C, Stimmel G (2012) Characterizing The Relationship Between Individuals with Mental Health Conditions and Community Pharmacists. CPNP and NAMI Organization.

12. Lancaster J, Douglass M, Gonyeau M, Wong A, Woolley A, et al. (2013) Providers' Perceptions of Student Pharmacists on Inpatient General Medicine Practice Experiences. Am J Pharm Educ77: 26. [Crossref]

13. Moyer VA, LeFevre M, Calonge N (2016) Is It Time for the USPSTF to Inform-But Not Determine-Coverage? Ann Intern Med 165: 876-877. [Crossref]

14. Cornally N, Coffey A, Daly E, McGlade C, Weathers E, et al.(2016) Measuring Staff Perception of End-of life Experience of Older Adults in Long-term Care. ApplNurs Res 30: 245-251.[Crossref]

15. McNamara KP, George J, O'Reilly SL, Jackson SL, Peterson GM, et al. (2010) Engaging Community Pharmacists in The Primary Prevention of Cardiovascular Disease. BMC Health Serv Res 10: 264. [Crossref]

16. Riley AC, Lee EJ (2015) Understanding of Medication Directions: Assessment of Native Korean Older Adults. Consult Pharm 30: 671-677. [Crossref]

Copyright: (C2017 Osisanya F. This is an open-access article distributed under the terms of the Creative Commons Attribution License, which permits unrestricted use, distribution, and reproduction in any medium, provided the original author and source are credited. 